\title{
Band Formation in Mixtures of Oppositely Charged Colloids Driven by an ac Electric Field
}

\author{
Teun Vissers, ${ }^{*}$ Alfons van Blaaderen, and Arnout Imhof ${ }^{\dagger}$ \\ Soft Condensed Matter, Debye Institute for Nanomaterials Science, Utrecht University, \\ Princetonplein 5, 3584 CC, Utrecht, The Netherlands \\ (Received 15 February 2011; published 3 June 2011)
}

\begin{abstract}
We present experiments on pattern formation in a Brownian system of oppositely charged colloids driven by an ac electric field. Using confocal laser scanning microscopy we observe complete segregation of the two particle species into bands perpendicular to a field of sufficient strength when the frequency is in a well-defined range. Because of its Brownian nature the system spontaneously returns to the equilibrium mixture after the field is turned off. We show that band formation is linked to the time scale associated with collisions between particles moving in opposite directions.
\end{abstract}

PACS numbers: 82.70.Dd, 47.54.-r, 89.75.Kd

Pattern formation is found on many different length scales and as a consequence is studied in molecular systems [1,2], geology [3], the animal kingdom [4,5], granular systems [6], and colloids [7]. Granular and colloidal systems are especially interesting since they can be studied in real space on the single-particle level due to their slow dynamics and relatively large size compared to atoms and molecules [8,9]. A visually compelling case of pattern formation is found in binary granular systems containing two different particles species that segregate when the mixture is subjected to mechanical agitation [10-12]. Recently, it was shown that periodically sheared granular suspensions [13] can undergo an out-of-equilibrium phasetransition from a dynamic fluctuating state into a quiescent "absorbing state", which can also be found in epidemics and on the surface of certain heterogeneous catalysts [14].

Computer simulations have recently identified binary mixtures of colloidal particles driven in opposite directions by an external field as an interesting test bed for pattern formation in complex systems $[15,16]$. Such colloidal systems are all the more interesting because they are thermal systems: Brownian motion allows any induced nonequilibrium state to spontaneously relax back to the equilibrium mixture, and presents pattern formation as a competition between field-induced ordering and thermal disordering. Such a binary colloidal system is hard to realize in experiment, however, because of strict requirements placed on a sufficiently strong interaction with the driving field and the thermodynamic stability of the mixture. Nevertheless, we recently succeeded in developing a system of oppositely charged colloids that can be studied in 3D real space when exposed to an external electric field. We performed a combined experimental and Brownian dynamics simulation study, which showed that as a result of collisions between particles moving in opposite directions, the different particle species segregate into lanes parallel to the field [17]. We also demonstrated that the fraction of particles in a lanelike environment increased monotonically with field strength. Once particles were in a lane, fluctuations perpendicular to the field axis decreased and the electrophoretic mobility increased with respect to particles not in a lane.

Here, we employ this binary mixture to investigate large scale pattern formation into bands perpendicular to an applied AC electric field. The patterns found are reminiscent of those seen in granular systems [10,12], but contrary to those systems, relax back fully to the equilibrium mixture after the field is turned off. Moreover, changing field strength or frequency switches reversibly between banded, laned, or fully mixed states. Our study also identifies the origin of the banded state as arising from the field reversing repeatedly before a steady state of lanes can form. We believe this is the first demonstration of such behavior, and that our work provides important clues into the mechanism of pattern formation in driven systems.

We used binary dispersions of sterically stabilized polymethylmethacrylate (PMMA) particles. The particles were synthesized by dispersion polymerization [18] and covalently labeled with the fluorescent dye 7-nitrobenzo-2oxa-1,3-diazol (NBD, color-coded "green") or rhodamine isothiocyanate (RITC, "red"). After washing the colloids several times with hexane, and dispersing them in a refractive-index matching mixture of 72.8 wt $\%$ cyclohexylbromide and 27.2 wt \% cis-decahydronaphthalene, the dispersion was transferred into a capillary (Vitrocom) with two $50 \mu \mathrm{m}$ (diameter) nickel alloy wires (Goodfellow) threaded along the length direction serving as electrodes. To induce opposite charges on the particles and screen the electrostatic interactions, the solvent mixture contained tetrabutylammonium bromide (TBAB) salt. From the measured conductivity, and taking into account the partial dissociation of TBAB, we estimated the inverse Debye-Hückel screening length $\kappa^{-1}$.

The capillary was sealed with UV-glue (Norland optical adhesive) that was cured ( $\lambda=350 \mathrm{~nm}$, UVGL-58 UV lamp, UVP) while shielding the capillary from UV light to prevent partial decomposition of the solvent [19]. A sketch of the setup is given in supplemental Fig. 1. To supply the electric driving, we used a function generator 


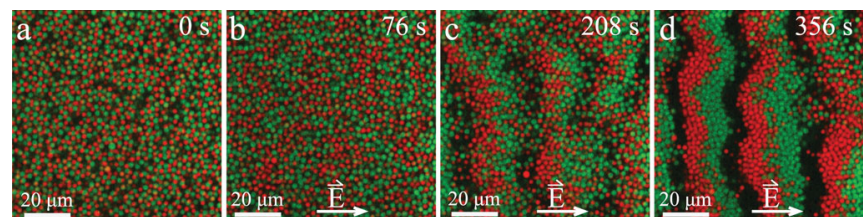

FIG. 1 (color). Band formation of oppositely charged colloids with a diameter of ca. $2.5 \mu \mathrm{m}$ in an ac electric field with strength $|E|=17.5 \mathrm{~V} / \mathrm{mm}$, and frequency $f=0.02 \mathrm{~Hz}$. (a) No field is applied and the mixture is isotropic, (b) locally bands start to form after $76 \mathrm{~s}$, (c) the bands become more pronounced, and (d) the bands are clearly delineated.

(Agilent, Model 3312 OA) and wideband amplifier (Krohn-Hite, Model 7602M). The electric field was applied in the $\mathbf{x}$ direction. Measurements were performed in a region approximately in the middle between the electrodes. All images $(1024 \times 512$ or $512 \times 256$ pixels $)$ were recorded on a Leica SP2 confocal microscope at 1 to 2 frames per second. Particle positions were determined using a tracking algorithm similar to that of Crocker et al. [20].

Figure 1 shows band formation in a binary system containing negatively ("green", $\sigma_{G}=2.57 \mu \mathrm{m}$, polydispersity 3\%) and positively charged particles ("red", $\sigma_{R}=2.48 \mu \mathrm{m}$, polydispersity $4 \%$ ) driven by an AC electric field with strength $|E|=17.5 \mathrm{~V} / \mathrm{mm}$ and frequency $f=0.02 \mathrm{~Hz}$. The average volume fraction was $\phi_{G}=$ $\phi_{R}=0.14$ for both particle species. From the electrophoretic mobilities in the mixed concentrated suspension $\mu_{G}=-3.6 \times 10^{-11} \mathrm{~m}^{2} / \mathrm{Vs}$ and $\mu_{R}=2.9 \times 10^{-11} \mathrm{~m}^{2} / \mathrm{Vs}$ and the concentration of TBAB salt (ca. $2 \mu \mathrm{M}$ ), we estimate the particle charges to be $Z_{G} e \approx-102 e$ for the green and $Z_{R} e \approx 78 e$ for the red particles (supplemental in [17], [21]). The screening length $\kappa^{-1} \approx 550 \mathrm{~nm}$. In each cycle of the field the bands grew as particles moving in opposite directions collided and, as a result, were pushed in the direction perpendicular to the field (see also supplemental video 1 [22]). It was not possible to dissolve these bands by changing the frequency of the field.

However, we found that for smaller-and thus more mobile-micron-sized particles we could quickly remix the suspension and perform multiple experiments in the same electric cell. For this, we used the same colloidal dispersion as in [17], containing negatively charged particles ("green") with a diameter $\sigma_{g}=1.06 \mu \mathrm{m}$ and a polydispersity of $6 \%$ and positively charged particles ("red") with diameter $\sigma_{r}=0.91 \mu \mathrm{m}$ and a polydispersity of $7 \%$. The average volume fraction was $\phi_{g}=\phi_{r}=0.09$ for both particle species. The particle charges, as estimated in [17], were approximately $Z_{g} e \approx-42 e$ for the green, and $Z_{r} e \approx 67 e$ for the red particles. The screening length $\kappa^{-1} \approx 170 \mathrm{~nm}$. The mixed dispersion is shown in Fig. 2(a). When an ac electric field with a field strength of $104 \mathrm{~V} / \mathrm{mm}$ and of frequency $1.5 \mathrm{~Hz}$ (block) was applied - much higher than for the $2.5 \mu \mathrm{m}$ particles—bands formed within (a few) seconds [Fig. 2(b)]. A single band obtained a characteristic width of a few particle diameters. The bands were found to meander and change in thickness over time. Moreover, their shape depended on the applied field strength, frequency and local volume fraction. As can be seen in Fig. 2(b), the bands were often tilted in an angle that was not exactly $\pi / 2$ with respect to the oscilatory force. We note that such a "tilted band state", as an intermediate state between axial segregation and laning, was also found in [16]. We observed that reducing the frequency by a factor of 15 [from 1.5 to $0.1 \mathrm{~Hz}$ in Fig. 2(c)] resulted in complete dissolution of the band-patterns in about $10 \mathrm{~s}$. After the bands had been erased, lane formation took over [Fig. 2(d)]. Turning off the field after this step yielded back a mixed system [similar to Fig. 2(a)]. The whole cycle took $135 \mathrm{sec}$ to complete and is also shown in supplemental video 2 [22].
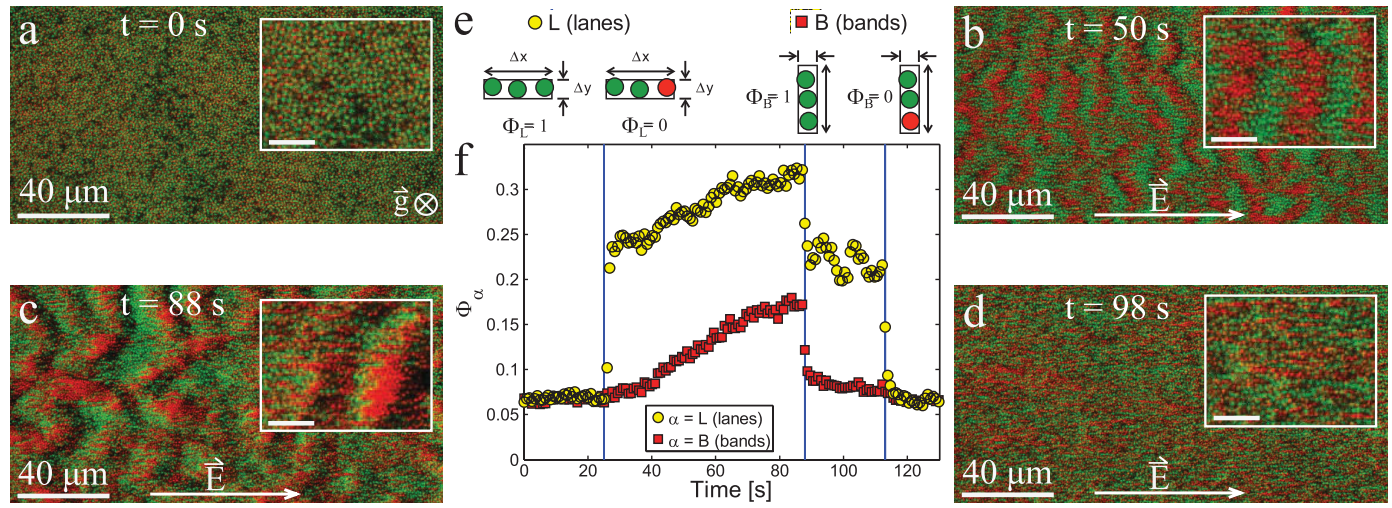

FIG. 2 (color). Typical confocal images of band formation for micron-sized particles. (a) The initial mixed binary system, (b) band formation $25 \mathrm{sec}$ after a field with field strength $|E|=104 \mathrm{~V} / \mathrm{mm}$ and frequency $f=1.5 \mathrm{~Hz}$ had been switched on, (c) bands break up just after the frequency had been changed to $f=0.1 \mathrm{~Hz}$, and (d) lanes $10 \mathrm{~s}$ after the frequency had been changed to $f=0.1 \mathrm{~Hz}$. For each image, a local close-up is shown. After the field had been turned off, a mixed dispersion as shown in (a) was reobtained (not shown here). (e) Examples of the lane- and band-order parameter. (f) The order parameters $\Phi_{B}$ (red $\square$ ) and $\Phi_{L}$ (yellow $\bigcirc$ ) during the cycle. The blue vertical lines in the diagrams denote changes in field strength or frequency. The scale bars in the insets are $10 \mu \mathrm{m}$. Larger versions of images (a)-(d) are shown in supplemental Fig. 3. 
The "erasing" step was not necessary to obtain complete remixing; when the field was turned off with the bands still present, the system also remixed by diffusion in approximately 20 min (supplemental Fig. 2).

Because of sedimentation, the colloidal volume fraction $\phi(z)$ in our system depended on the depth $z$ in the capillary [17]. In the lower half of the capillary channel, the volume fraction was sufficiently high $\left(\phi_{g+r} \approx 0.21-0.24\right)$ for the formation of bands. In the upper half region of the channel, the volume fraction was substantially lower, and no band formation was observed for the field strengths that were applied here. The band-formation images were recorded close to the lower theoretical stationary layer, where there is no fluid movement due to electro-osmosis.

To quantify the degree of band formation, we evaluated the fraction of particles that have a bandlike environment [see Fig. 2(e)]. To do this, all particles within the optical slice were projected onto the $(x y)$ plane. To detect bands perpendicular to the field axis, a rectangle of length $\Delta x=1.2 \sigma$ along the driving field and width $\Delta y=4.0 \sigma$ perpendicular to it was constructed around each particle $i$. To detect structures aligned with the field axis (lanelike), this rectangle was simply rotated 90 degrees. We define $\Phi_{i}^{\alpha}=1$ when all other particles in this rectangle are of the same species as particle $i$, otherwise, $\Phi_{i}^{\alpha}=0$. In the rare case that particle $i$ is alone, $\Phi_{i}^{\alpha}=0$. The averaged order parameter is given by $\Phi_{\alpha}=\frac{1}{N} \sum_{i=1}^{N} \Phi_{i}^{\alpha}$ and represents the fraction of particles that are in a lanelike $(\alpha=L)$ or bandlike $(\alpha=B)$ environment.

In Fig. 2(f), the time evolution of $\Phi_{L}$ and $\Phi_{B}$ is shown for the experiment depicted in Fig. 2(a)-2(d). Since a small probability exists for particles to have a lane- or bandlike configuration by chance, both order parameters had a small, but nonzero value for $|E|=0$. Therefore, only the excess of $\Phi$ compared to its zero-field reference value is a measure for lane or band formation.

After the field was turned on $(t=26 \mathrm{~s}), \Phi_{L}$ made a jump, indicating that lanes formed almost instantaneously.
As was shown in [17], the lanes would have remained in a steady state if the field had not reversed. The monotonic rise of $\Phi_{B}$ shows that the number of particles in a bandlike environment grew steadily in time from this point on. In addition, $\Phi_{L}$ further increased during the growth stage. When the frequency was lowered from 1.5 to $0.1 \mathrm{~Hz}, \Phi_{B}$ rapidly decreased as all the bands were destroyed within one cycle of the alternating field. $\Phi_{L}$ also decayed fast to the same value as immediately after the $1.5 \mathrm{~Hz}$ field had been turned on. When the field was finally turned off, both order parameters returned to their original value on $t=0$.

In Fig. 3(a), we study the response to changes in field strength while keeping the frequency fixed at $4.0 \mathrm{~Hz}$ (block wave). For each consecutive change, there was a corresponding swift response in $\Phi_{L}$, indicating that on the time scale of the measurements (ca. $0.5 \mathrm{~s}$ per frame), the number of lanes directly followed the electric field. The growth of bands perpendicular to the field, indicated by an increase in $\Phi_{B}$, was slower and started only when the field was increased from $103 \mathrm{~V} / \mathrm{mm}$ to $122 \mathrm{~V} / \mathrm{mm}$. After $50 \mathrm{~s}, \Phi_{B}$ leveled off as the system approached a steady state. When the field strength was further increased to $135 \mathrm{~V} / \mathrm{mm}$, $\Phi_{B}$ increased again until ca. $25 \%$ of the particles were in a bandlike environment before leveling off again. Subsequently, the field strength was lowered to $|E|=$ $51 \mathrm{~V} / \mathrm{mm}$, resulting in the dissolution of the bands. The fact that the bands did not immediately dissolve indicates some degree of hysteresis. Lowering the frequency to $f=0.1 \mathrm{~Hz}$ quickly remixed the system.

To study the effect of the field frequency on the band formation, different frequencies were applied at a driving field of $|E|=108 \mathrm{~V} / \mathrm{mm}$ [peak, a sine function was used in Fig. 3(b)]. At $f=0.8 \mathrm{~Hz}, f=0.6 \mathrm{~Hz}$ and $f=0.5 \mathrm{~Hz}$ it took the system increasingly longer times, 47, 115 and $157 \mathrm{~s}$, respectively, to reach a comparable degree of band formation. At a frequency $f=0.1 \mathrm{~Hz}$ (block wave) we did not observe band formation for this system. Moreover,
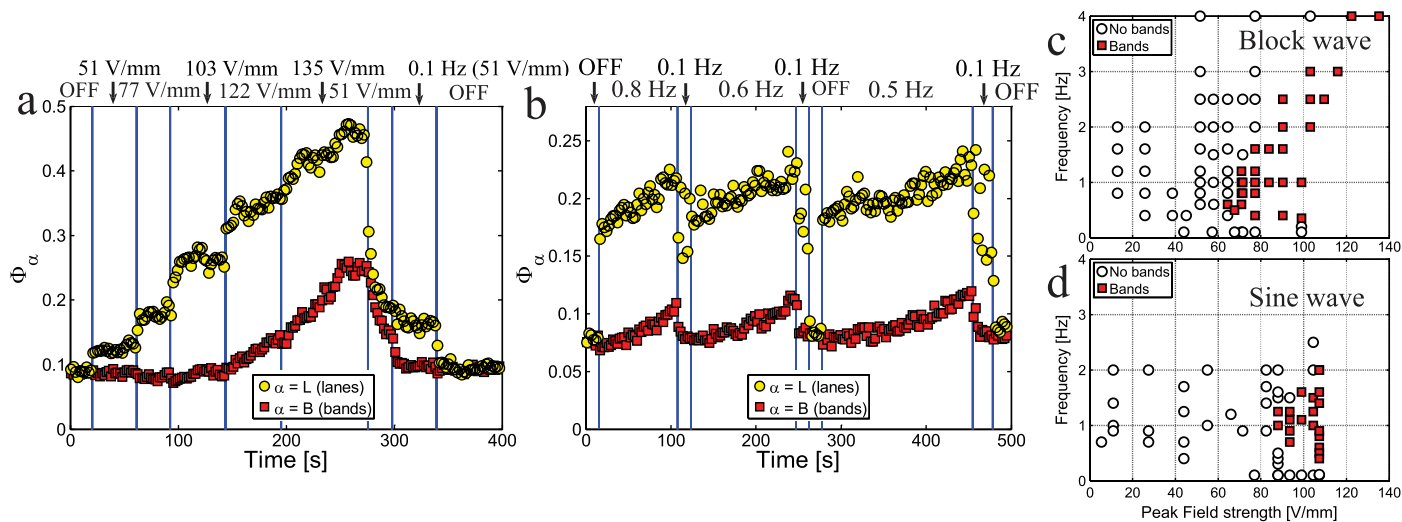

FIG. 3 (color online). Dependence of band formation on field strength and frequency for the micron-sized particles. (a),(b) Order parameters $\Phi_{B}(\square)$ and $\Phi_{L}(\bigcirc)$ as a function of time for two different cases. (a) The field strength was increased stepwise at a frequency of $4.0 \mathrm{~Hz}$ (block wave). (b) At fixed field strength (peak $108 \mathrm{~V} / \mathrm{mm}$ ), the frequency (sine wave) was changed as indicated on top of the graph. (c),(d) Out-of-equilibrium phase diagram, using the block or sine wave field. Circles denote that no band formation was observed. Red squares indicate band formation. 
existing bands always disappeared if the frequency was lowered to $f=0.1 \mathrm{~Hz}$.

These measurements show that lane formation increases continuously with field strength. The transition towards band formation, on the other hand, requires a minimum field strength and frequency to occur. We can therefore draw up a nonequilibrium diagram demarcating the region where bands formed. Figure 3(c) and 3(d) summarizes the results of multiple measurements for both a block and sine function. When $\Phi_{B}$ exceeded its zero-field value above the noise, bands also became visually apparent, and the point is depicted with a red square. Points for which no band formation was visible are marked with a circle. Note that a square does indicate that at least some degree of band formation was ongoing, but does not necessarily mean that a steady state was always reached within the time of observation. For both the sine and the block wave, there was both a minimum field strength $E_{c}$ and a minimal frequency $f_{c}$ required to induce band formation. For the sine function, this critical field strength was higher than for the block function. Note that the integral over a half-cycle of the field differs between the sine and the block function by a factor of $\pi / 2$ and roughly corresponds to the difference in $E_{c}$, indicating that the driving distance per period is likely a key parameter.

Above $E_{c}$, the frequency range where bands appear widens, to both lower and higher frequencies. Although we did not directly study band formation as a function of volume fraction, this behavior shows some similarity with the critical behavior observed in a shaken (quasi-2D) binary granular system, where the formation time of bands diverges near a critical surface coverage fraction [11].

In [17], it was found that a steady state consisting of lanes was reached within approximately $1 \mathrm{~s}$ after applying a dc electric field $(|E|=120 \mathrm{~V} / \mathrm{mm})$ to the same system of $1 \mu \mathrm{m}$ particles. This can be seen from the rapid rise of the lane order parameter in Fig. 2(f). Before this steady state was reached, we observed that fluctuations perpendicular to the field temporarily increased in the first half second, since oppositely charged particles had to move around each other as a result of mutual collisions. From Fig. 3(c) we can see that bands start to form at frequencies higher than ca. $0.5 \mathrm{~Hz}$. Therefore, periodically reversing the field for frequencies higher than this keeps the number of collisions high, forcing the particles to keep rearranging until they are surrounded by particles of the same kind. This favors the banded state, where only particles at interfaces still undergo collisions with particles of the other kind. When the frequency becomes too high the particle amplitude, which is of order $\mu E / f$, becomes too small to induce a sufficient number of collisions for bands (or lanes) to form and the system remains mixed. Finally, we note that if the filling fraction is sufficiently high, it is predicted that bands can form in dc fields as well $[23,24]$. This socalled "jamming" was indeed observed experimentally for oppositely charged particles [25].
Our experiments reveal a new type of pattern formation in a nonequilibrium system of oppositely charged colloidal particles. In addition to the formation of lanes parallel to a dc electric field, we demonstrate that bands form perpendicular to an $\mathrm{AC}$ field with the proper strength and frequency. Our data suggest that band formation occurs when the field direction reverses on a time scale on which lanes are still being established. The increased number of collisions between particles moving in opposite directions leads to enhanced lateral motion, facilitating the formation of bands. An out-of-equilibrium phase diagram was constructed that shows the regime in which band-formation takes place. Changing field strength or frequency switches reversibly between banded, laned, or fully mixed states.

We gratefully acknowledge Adam Wysocki, Hartmut Löwen, and Michiel Hermes for useful discussions. We thank Peter Helfferich for technical support and Johan Stiefelhagen for his help with the assembly of the electric cells and, along with Didi Derks, for particle synthesis. NWO-CW and EU (NanoDirect, CP-FP-213948-2) are acknowledged for financial support.

*t.vissers@uu.nl

†a.imhof@uu.nl

[1] M. C. Cross and P. C. Hohenberg, Rev. Mod. Phys. 65, 851 (1993).

[2] G. I. Sivashinsky, Annu. Rev. Fluid Mech. 15, 179 (1983).

[3] M. A. Kessler and B. T. Werner, Science 299, 380 (2003).

[4] I. D. Couzin and N. R. Franks, Proc. R. Soc. B 270, 139 (2003).

[5] M. Moussaid et al., Topics in Cognitive Sci. 1, 469 (2009).

[6] I. S. Aranson and L. S. Tsimring, Rev. Mod. Phys. 78, 641 (2006).

[7] C. P. Royall et al., Phys. Rev. Lett. 98, 188304 (2007).

[8] A. van Blaaderen and P. Wiltzius, Science 270, 1177 (1995).

[9] V. Prasad, D. Semwogerere, and E. R. Weeks, J. Phys. Condens. Matter 19, 113102 (2007).

[10] T. Mullin, Phys. Rev. Lett. 84, 4741 (2000).

[11] P. M. Reis and T. Mullin, Phys. Rev. Lett. 89, 244301 (2002).

[12] P. Sánchez, M. R. Swift, and P. J. King, Phys. Rev. Lett. 93, 184302 (2004).

[13] L. Corte et al., Nature Phys. 4, 420 (2008).

[14] R. Dickman, Physica (Amsterdam) 306A, 90 (2002).

[15] H. Löwen and J. Dzubiella, Faraday Discuss. 123, 99 (2003).

[16] A. Wysocki and H. Löwen, Phys. Rev. E 79, 041408 (2009).

[17] T. Vissers et al., Soft Matter 7, 2352 (2011).

[18] G. Bosma et al., J. Colloid Interface Sci. 245, 292 (2002).

[19] D. El Masri et al., Soft Matter 7, 3462 (2011).

[20] J. C. Crocker and D. G. Grier, J. Colloid Interface Sci., 179, 298 (1996).

[21] T. Vissers et al., J. Colloid Interface Sci. (to be published).

[22] See supplemental material at http://link.aps.org/ supplemental/10.1103/PhysRevLett.106.228303.

[23] K. E. Bassler et al., Europhys. Lett. 24, 115 (1993).

[24] D. Helbing, I. J. Farkas, and T. Vicsek, Phys. Rev. Lett. 84, 1240 (2000).

[25] M. E. Leunissen et al., Nature (London) 437, 235 (2005). 